\title{
Local stability of self-gravitating fluid disks made of two components in relative motion
}

\author{
G. Bertin ${ }^{1}$ and A. Cava ${ }^{1,2}$ \\ 1 Università degli Studi di Milano, Dipartimento di Fisica, via Celoria 16, 20133 Milano, Italy \\ e-mail: giuseppe.bertin@unimi.it \\ 2 INAF - Osservatorio Astronomico di Padova, Vicolo dell'Osservatorio 5, 35122 Padova, Italy \\ e-mail: antonio.cava@oapd.inaf.it
}

Received 12 February 2006 / Accepted 4 August 2006

\section{ABSTRACT}

\begin{abstract}
Context. We consider a simple self-gravitating disk, made of two fluid components characterized by different effective thermal speeds and interacting with one another only through gravity; two-component models of this type have often been considered in order to estimate the impact of the cold interstellar medium on gravitational instabilities in star-dominated galaxy disks.

Aims. This simple model allows us to produce a unified description of instabilities in non-viscous self-gravitating disks, some originating from Jeans collapse, and others from the relative motion between the two components. In particular, the model suggests that the small streaming velocity between the two components associated with the so-called asymmetric drift may be the origin of instability for suitable non-axisymmetric perturbations.

Methods. The result is obtained by examining the properties of a local, linear dispersion relation for tightly wound density waves in such two-component model. The parameters characterizing the equilibrium model and the related dispersion relation allow us to recover as natural limits the cases, known in the literature, in which the relative drift between the two components is ignored.

Results. Dynamically, the instability is similar to (although gentler than) that known to affect counter-rotating disks. However, in contrast to the instability induced by counter-rotation, which is a relatively rare phenomenon, the mechanism discussed in this paper is likely to be rather common in nature.

Conclusions. We briefly indicate some consequences of the instability on the evolution of galaxy disks and possible applications to other astrophysical systems, in particular to protostellar disks and accretion disks.
\end{abstract}

Key words. galaxies: kinematics and dynamics - instabilities

\section{Introduction}

As clearly noted in investigations of the problem of spiral structure in galaxies, the dynamics of galaxy disks depends significantly on the mutual interaction between gas and stars. For the general issues related to the properties of waves and instabilities, such gas-star interactions have often been studied by referring to a very simple, idealized two-component model in which one distinguishes a "hot" component (meant to represent mostly the older stellar disk) from a "cold" component (representative of atomic and molecular hydrogen and young stars) interacting with each other only through gravity. In practice, calculations have been carried out by treating one component by means of the equations of stellar dynamics and the other by fluid equations, or, for simplicity, by treating both components by means of simple barotropic fluid models characterized by different effective thermal speeds; other investigations have implemented these concepts by means of suitable N-body simulations. It has thus been ascertained that even small amounts of gas can have significant influence on the Jeans stability of galaxy disks (e.g., see Lin \& Shu 1966; Lynden-Bell 1967; Graham 1967; Miller et al. 1970; Quirk 1971; Kato 1972; Jog \& Solomon 1984a,b; Sellwood \& Carlberg 1984; Bertin \& Romeo 1988, hereafter BR88).

Similar models have also been used to examine situations in which the two components are in relative motion with respect to one another. Among the earliest investigations, we may recall those by Sweet (1963), Lynden-Bell (1967),
Wilson \& Lynden-Bell (unpublished; as quoted in Lynden-Bell 1967), and Marochnik \& Suchkov $(1969,1974)$. Often in such early studies the "Population II" stars are treated as a nonrotating component, in a kinetic model, and, for simplicity, the geometry adopted is that of a homogeneous three-dimensional system (see also the extensive discussion of the interaction between gas and stars in the monograph by Saslaw 1985). Later on, similar studies were further motivated by the issue of disk-halo interaction (Mark 1976); so, in the latter investigations one component represents the rotating disk and the other the non-rotating or slowly rotating halo material. These analyses then brought out the possibility of a kind of two-stream or Kelvin-Helmholtz instability, thus beyond Jeans instability, with applications to the amplification of bending waves in relation to the problem of galaxy warps (Bertin \& Mark 1980; Bertin \& Casertano 1982; revisited by Nelson \& Tremaine 1995). With similar tools, others have explored the possibility of interactions between different gaseous components in galaxies (see Nelson 1976; Waxman 1979a,b).

More recently, renewed attention has been drawn to the study of instabilities excited in disks in relative motion (Sellwood \& Merritt 1994; Lovelace et al. 1997, hereafter LJH97; Hunter et al. 1997; Comins et al. 1997; Mandica 2001) by the discovery of the curious phenomenon of counter-rotation, noted in a number of disk galaxies (Rubin et al. 1992; Rix et al. 1992; Merrifield \& Kuijken 1994; Bertola et al. 1996; Jore et al. 1996; Prada et al. 1996; Thakar et al. 1997; Bettoni et al. 2001). 
Counter-rotation appears to be a relatively rare, and possibly short-lived phenomenon. Thus, such relative motion between two disk components is likely to be only rarely involved in the dynamics of galaxies. In contrast, in this paper we focus our attention on a potential source of instability that might be ubiquitous: because of the so-called asymmetric drift associated with the finite velocity dispersion of the hot component (e.g., see Bottema 1993), any disk of stars and gas would in general be characterized by small, but finite relative motion between the two components.

In this paper we will show that, in principle, the small velocity difference between two components associated with the asymmetric drift is a source of instabilities in the disk. This result will be obtained by inspection of a local, linear dispersion relation for tightly wound density waves derived from a simple two-fluid model in Sect. 2. In Sect. 3 we will study the conditions for the onset of the asymmetric-drift instability and its main characteristics. Finally, in Sect. 4 we will describe possible applications to different astrophysical contexts and draw the main conclusions of our paper. Given the fact that such asymmetricdrift velocity is small, for applications to galaxy disks, this paper is meant to offer an investigation preliminary to a fluid-kinetic analysis that is planned for the future.

While this work was being completed, we were reminded of an interesting study made in the context of the dynamics of protostellar disks (Weidenschilling 1977), about the viscous drag exerted by the gas of the protostellar disk on the population of planetesimals, which presents significant analogies with the mechanism addressed in this paper. We will briefly comment on this point in the conclusion section.

\section{The fluid model and the relevant dispersion relation}

In a rotating self-gravitating axisymmetric fluid disk at equilibrium the radial gravitational force is balanced by rotation, with a contribution from the pressure gradient:

$\Omega^{2}=\frac{1}{r \sigma} \frac{\mathrm{d} p}{\mathrm{~d} r}+\frac{1}{r} \frac{\mathrm{d} \Phi}{\mathrm{d} r}$,

where $\Omega, \sigma$, and $p$ are the equilibrium angular velocity, surface density, and pressure of the thin disk, and $\Phi$ is the total gravitational potential. For cool disks, the pressure gradient is small and generally neglected. Therefore, many studies of the dynamics of such disks simply refer to the angular frequency as defined by the relation $r \Omega_{0}^{2} \equiv \mathrm{d} \Phi / \mathrm{d} r$ and to the related epicyclic frequency defined by $\kappa_{0}^{2} \equiv 4 \Omega_{0}^{2}\left[1+(1 / 2) \mathrm{d} \ln \Omega_{0} / \mathrm{d} \ln r\right]$.

We now consider an infinitesimally thin, self-gravitating, two-component disk. In general, the two components are characterized by different equivalent acoustic speeds $c_{i}$ and surface densities $\sigma_{i}$. We label the two components by the subscripts $\mathrm{c}$ and $\mathrm{h}$ (in relation to their equivalent acoustic speeds, so that $c_{\mathrm{c}}<c_{\mathrm{h}}$ ). Similarly to other previous stability analyses, we assume that the only interaction between the components occurs via the gravitational field. In contrast with previous analyses that focused on the Jeans instability and assigned the same equilibrium angular velocity $\Omega_{0}$ to the two components (e.g., see BR88), in this paper we allow for the presence of relative motion between the two components, so that in general $\Omega_{\mathrm{c}} \neq \Omega_{\mathrm{h}}$, and, in turn, for a difference in the associated epicyclic frequencies $\kappa_{\mathrm{c}} \neq \kappa_{\mathrm{h}}$. The special limiting case in which $c_{\mathrm{c}} \longrightarrow c_{\mathrm{h}}$ and $\Omega_{\mathrm{c}} \longrightarrow-\Omega_{\mathrm{h}}$ thus corresponds to the case of counter-rotating components, a situation already discussed in the literature, as mentioned in the Introduction. We argue that, because of the different size of the relevant asymmetric drift dictated by Eq. (1) the angular velocities of the two components would in general be (although slightly) different even when the two components are not counter-rotating.

\subsection{Applicability of the two-fluid model}

The two interpenetrated non-viscous fluids studied in this paper are to be taken as the simplest model in which we can include several key factors (temperature, density, and velocity differences, within the non-trivial geometry of an inhomogenous thin layer) that determine the stability of a two-component self-gravitating disk. Some important features, such as finite thickness, present in real self-gravitating disks are not included. Therefore, for any specific application (for example, to the dynamics of galaxy disks) it will be necessary to check to what extent the basic approximations implicit in this model are justified and, if necessary, to extend the calculations to a more realistic (and thus more complicated) model. On the other hand, studying this simple model has the advantage of leading to a unified picture of a variety of instabilities that may affect a self-gravitating disk.

One particular point of concern that will emerge from the following analysis is related to the applicability of the two-fluid model to cases in which the relative speed between the two components is small. In fact, it is well known that, for a kinetic system made of two components in relative streaming, the fluid description becomes inadequate when the relative speed is small (e.g., see Lynden-Bell 1967; Krall \& Trievelpiece 1973). The instability noted in a two-fluid description may remain, but is usually modified because the underlying mechanism of instability is associated with an inverse Landau damping (i.e., the effects of wave-particle resonances) that only a kinetic description can properly describe. Thus, in view of applications to a disk made of stars and gas (a system that is neither fully kinetic nor fully fluid), the present study should be completed by investigating the mechanisms in a mixed fluid-kinetic model; this extension will be addressed in a separate paper.

\subsection{Local dispersion relation}

We now consider tightly wound linear density perturbations of the form $\sigma_{1 i}=\hat{\sigma}_{1 i} \exp \left[\mathrm{i}\left(-\omega t+m \theta+\int^{r} k \mathrm{~d} r\right)\right]$ under the WKB ordering $m /(r|k|)=O(\epsilon)$, with the epicyclic expansion parameter defined as $\epsilon=c_{\mathrm{h}} /\left(r \kappa_{\mathrm{h}}\right)$. We will take the azimuthal number $m$ to be positive, so that trailing disturbances are characterized by radial wave number $k>0$. To lowest order, the density response for each component (resulting from the relevant continuity and Euler equations) is given by

$\sigma_{1 i}=\sigma_{i} \frac{k^{2} \Phi_{1}}{\left(\omega-m \Omega_{i}\right)^{2}-\kappa_{i}^{2}-c_{i}^{2} k^{2}}$

Each component experiences the joint perturbed gravitational potential $\Phi_{1}$, which is calculated from the Poisson equation

$-|k| \Phi_{1}=2 \pi G\left(\sigma_{1 c}+\sigma_{1 h}\right)$

By combining the above equations to eliminate $\Phi_{1}$ (for a complete derivation see Cava 2004), we obtain the dispersion relation, which we write in dimensionless form as

$v^{4}-2 \eta v^{3}-A^{(2)} v^{2}+2 \eta A^{(1)} v+A^{(0)}=0$. 
Here the relevant coefficients $A^{(n)}=A^{(n)}\left(|\hat{k}| ; Q_{\mathrm{h}}^{2}, \alpha, \beta, \Delta\right)$ for $n \neq$ 1 and $A^{(1)}=A^{(1)}\left(|\hat{k}| ; Q_{\mathrm{h}}^{2}\right)$ are defined as

$A^{(2)}=1+A^{(1)}+\Delta-\alpha|\hat{k}|+\frac{1}{4} Q_{\mathrm{h}}^{2} \beta \mid \hat{k}^{2}$,

$A^{(1)}=1-|\hat{k}|+\frac{1}{4} Q_{\mathrm{h}}^{2}|\hat{k}|^{2}$,

$$
\begin{aligned}
A^{(0)}= & (1+\Delta) A^{(1)}-\alpha|\hat{k}|+\frac{1}{4} Q_{\mathrm{h}}^{2} \beta|\hat{k}|^{2} \\
& -\frac{1}{4} Q_{\mathrm{h}}^{2}(\alpha+\beta)|\hat{k}|^{3}+\frac{1}{16} \beta Q_{\mathrm{h}}^{4}|\hat{k}|^{4} .
\end{aligned}
$$

Here $\hat{k}=2 \pi G \sigma_{h} k / \kappa_{h}^{2}$ and $v=\left(\omega-m \Omega_{h}\right) / \kappa_{h}$ are the dimensionless radial wavenumber and the dimensionless Doppler-shifted frequency, respectively. The quantity $Q_{\mathrm{h}}=c_{h} \kappa_{h} /\left(\pi G \sigma_{h}\right)$ is the axisymmetric stability parameter relative to the hot component.

Thus, with respect to the one-component stability analysis (which is uniquely controlled by the axisymmetric stability parameter), the dispersion relation depends on four additional dimensionless parameters $\alpha, \beta, \Delta$, and $\eta$. For the first two (positive definite) parameters we follow the definition of BR88, i.e. $\alpha=\sigma_{\mathrm{c}} / \sigma_{\mathrm{h}}$ and $\beta=c_{\mathrm{c}}^{2} / c_{\mathrm{h}}^{2}<1$ are the relative density and "temperature" ratios. The two new parameters are defined in terms of the angular velocity ratio $\hat{\delta} \equiv \Omega_{\mathrm{c}} / \Omega_{\mathrm{h}}$ (which, for simplicity, was taken to be constant in the derivation of Eq. (4)) as $\eta=m(\hat{\delta}-1)\left(\Omega_{\mathrm{h}} / \kappa_{h}\right)$ and $\Delta=\hat{\delta}^{2}-\eta^{2}-1$; therefore, in the limit $\Omega_{\mathrm{c}} \longrightarrow \Omega_{\mathrm{h}}$ we have $\Delta \longrightarrow 0$ and $\eta \longrightarrow 0$. Note that $\eta$ vanishes for axisymmetric perturbations $(m=0)$ even when the two components are in relative motion $(\hat{\delta} \neq 1)$. Since we ascribe the difference in angular velocity to the different weight of the pressure term in Eq. (1), we expect the cooler component to rotate faster, so that in general $|\hat{\delta}|>1$.

\subsection{Recovery of known limiting cases}

For $\hat{\delta}=1$ the two components are corotating exactly, which is the case considered by BR88, while for $\hat{\delta}=-1$ the components are counter-rotating, which corresponds to the case considered by LJH97 (who, at variance with the present two-fluid description, considered a mixed fluid-kinetic model and a kinetickinetic model).

The one-component dispersion relation $v^{2}=A^{(1)}\left(|\hat{k}| ; Q_{\mathrm{h}}^{2}\right)$ is recovered by taking the limit $\beta \longrightarrow 1, \hat{\delta} \longrightarrow 1$ (so that $\eta \longrightarrow 0$ and $\Delta \longrightarrow 0$ ), and by letting $\alpha$ become vanishingly small; the second, odd branch (see Sect. 2.2 in BR88) of the dispersion relation then reduces to the condition $v^{2}=1+(1 / 4) Q_{\mathrm{h}}^{2}|\hat{k}|^{2}$ for modified sound waves in the "gas tracer". For a two-component system the limit of exactly corotating fluids is obtained by letting $\eta=0$ and $\Delta=0$ so that the dispersion relation reduces to $v^{4}-A_{0}^{(2)} v^{2}+A_{0}^{(0)}=0$, with $A_{0}^{(0)}\left(|\hat{k}| ; Q_{\mathrm{h}}^{2}, \alpha, \beta\right)=A^{(1)}-$ $\alpha|\hat{k}|+(1 / 4) Q_{\mathrm{h}}^{2} \beta|\hat{k}|^{2}-(1 / 4) Q_{\mathrm{h}}^{2}(\alpha+\beta)|\hat{k}|^{3}+(1 / 16) \beta Q_{\mathrm{h}}^{4}|\hat{k}|^{4}$; thus the marginal stability curve at the basis of the analysis of BR88 (see their Eq. (14)) is recovered by setting $A_{0}^{(0)}=0$.

\section{The asymmetric-drift instability}

\subsection{Axisymmetric disturbances: reduction to standard Jeans stability conditions}

We first consider the case of axisymmetric disturbances, that is the case of $\eta=0$, with $\Delta=\Delta_{0} \equiv \hat{\delta}^{2}-1>0$. Here the dispersion relation reduces to $v^{4}-A^{(2)} v^{2}+A^{(0)}=0$. Therefore, the marginal stability condition is given by $A^{(0)}=0$. From Eq. (7) it is clear that, even if $\Delta \neq 0$, the condition is the same as for the $\Delta=0$ case, provided the relevant parameters $\alpha$ and $\beta$ are suitably rescaled. In fact, if we define $\alpha^{\prime}=\alpha /\left(1+\Delta_{0}\right)$ and $\beta^{\prime}=\beta /\left(1+\Delta_{0}\right)$, we find that the condition $A^{(0)}\left(|\hat{k}| ; Q_{\mathrm{h}}^{2}, \alpha, \beta, \Delta_{0}\right)=0$ is equivalent to the condition $A_{0}^{(0)}\left(|\hat{k}| ; Q_{\mathrm{h}}^{2}, \alpha^{\prime}, \beta^{\prime}\right)=0$. Therefore, for axisymmetric disturbances the stability condition is the same as for the standard Jeans instability in two-component disks. In particular, local stability at all wavelengths is guaranteed when $Q_{\mathrm{h}} \geq \bar{Q}\left(\alpha^{\prime}, \beta^{\prime}\right)$, which replaces the local axisymmetric stability condition for one-component fluid disks $Q \geq 1$; the function $\bar{Q}=\bar{Q}(\alpha, \beta)$ is illustrated in Fig. 5 of BR88. Note that, in general, for $\Delta_{0}>0$ we have $\bar{Q}\left(\alpha^{\prime}, \beta^{\prime}\right)<\bar{Q}(\alpha, \beta)$, which corresponds to a stabilizing effect on $m=0$ perturbations. We should also point out that the rescaling of $\alpha$ and $\beta$ that has allowed us to make use of the marginal stability condition described in BR88 does not correspond to exactly mapping the roots of the dispersion relation with $\hat{\delta}>1$ into those of the case with $\hat{\delta}=1$.

\subsection{Instability for non-axisymmetric perturbations at small values of $\eta$}

We now refer to the case when the relative motion between the two components is small, that is $\eta \ll 1$, with $\Delta=\Delta_{0}-$ $\eta^{2}<\Delta_{0}$, and consider an equilibrium case that is marginally stable with respect to the Jeans instability, so that $Q_{\mathrm{h}}=$ $\bar{Q}\left(\alpha /\left(1+\Delta_{0}\right), \beta /\left(1+\Delta_{0}\right)\right)$. The "most dangerous perturbations" are those with wavenumber close to $|\bar{k}|$, defined by the relation $A_{0}^{(0)}\left(|\bar{k}| ; \bar{Q}^{2}, \alpha /\left(1+\Delta_{0}\right), \beta /\left(1+\Delta_{0}\right)\right)=0$; close to $|\bar{k}|$, the quantity $A^{(0)}$ is expected to be negative and small, because $\Delta<\Delta_{0}$. In addition, we expect $A^{(1)}>0$ (the last relation follows from the fact that the presence of a second component is known to have a destabilizing effect on axisymmetric perturbations, i.e. $\left.\bar{Q}\left(\alpha^{\prime}, \beta^{\prime}\right)>1\right)$. As to the value of the frequency for such conditions close to marginal stability, we expect it to be also small, and thus we can approximate the relevant dispersion relation as a quadratic

$-A^{(2)} v^{2}+2 \eta A^{(1)} v+A^{(0)} \sim 0$.

Therefore, in the wavenumber range close to $|\bar{k}|$ in which

$\eta^{2}\left(A^{(1)}\right)^{2}+A^{(2)} A^{(0)}<0$

we find a pair of complex-conjugate solutions one of which corresponds to instability, with dimensionless growth rate $v_{\mathrm{I}}=$ $O(\eta)$. The real part of the frequency for these complex solutions is given by

$v_{R} \sim \eta \frac{A^{(1)}}{A^{(2)}}<\eta$,

which, as anticipated, corresponds to a pattern speed $\Omega_{p} \equiv \omega_{R} / m$ in the range $\Omega_{\mathrm{h}}<\Omega_{p}<\Omega_{c}$.

These facts are illustrated in Fig. 1 , where we compare the dispersion relation in the vicinity of marginal stability from the exact (Eq. (4)) and from the "reduced" (Eq. (8)) expression, showing that the approximate quadratic relation can be accurate even for relatively large values of $\eta$. In Fig. 2 we also illustrate the behavior of the coefficients $A^{(0)}$ and $A^{(1)}$, in line with the arguments given above to derive the onset of the instability analytically. 

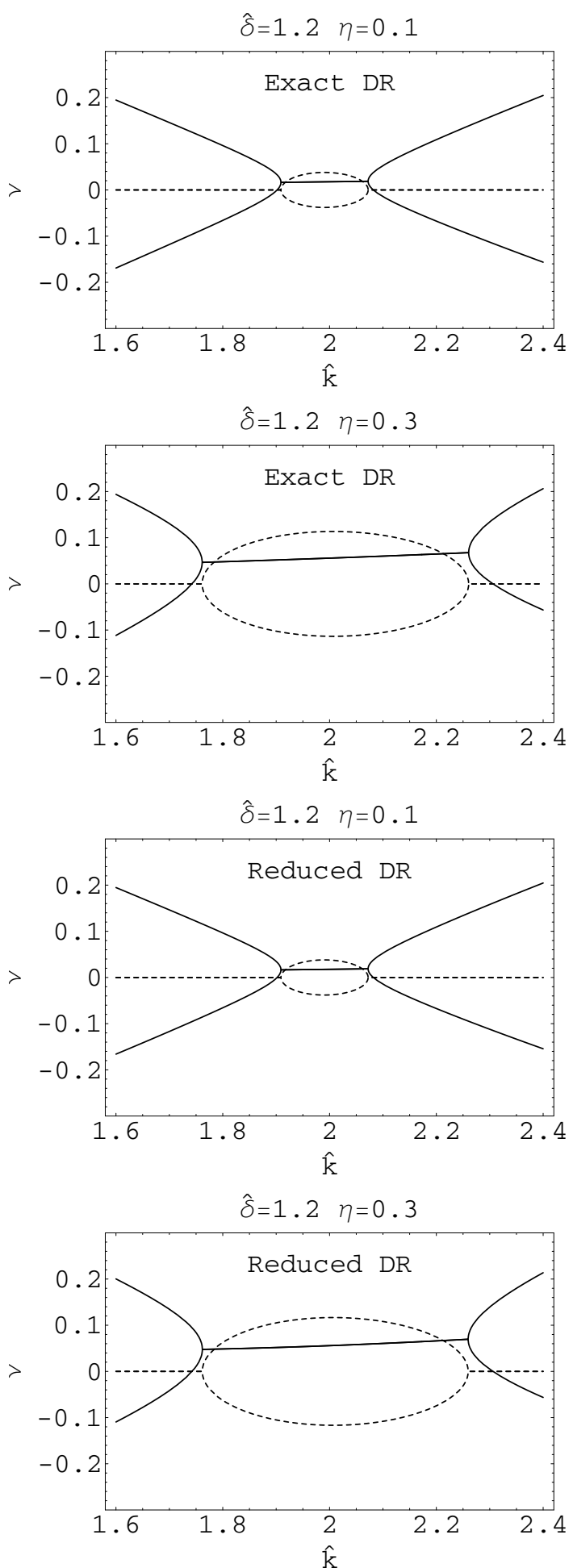

Fig. 1. The dispersion relation in the vicinity of marginal stability from the exact (Eq. (4); top frames) and from the "reduced" (Eq. (8); bottom frames) expression, for two cases characterized by $\hat{\delta}=1.2, \eta=0.1$ and $\hat{\delta}=1.2, \eta=0.3$. In each frame the real (solid lines) and imaginary (dashed lines) part of $v$ are given as a function of the dimensionless wavenumber $\hat{k}$. The other parameters have been set to the following values: $\alpha=0.1, \beta=0.1, Q_{\mathrm{h}}=\bar{Q}\left(\alpha / \hat{\delta}^{2}, \beta / \hat{\delta}^{2}\right) \approx 1.14$. The "reduced" dispersion relation turns out to be accurate even for $\eta=0.3$.

\subsection{Non-axisymmetric perturbations studied in the general case}

At this point we can move on to study the general case, when the new parameters $\Delta$ (or $\hat{\delta}$ ) and $\eta$ that appear in the dispersion

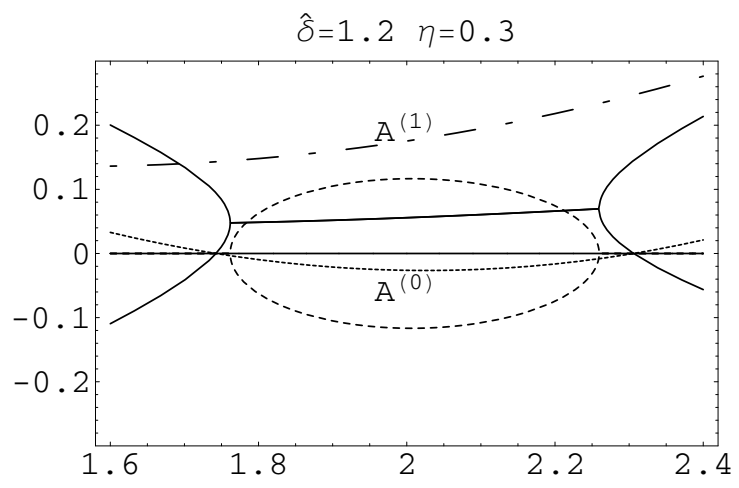

Fig. 2. Illustration of the generation of the unstable roots. Superposed to the dispersion relation shown in the second frame of Fig. 1 (for the case $\hat{\delta}=1.2, \eta=0.3$, with $\alpha=0.1, \beta=0.1, Q_{\mathrm{h}} \approx 1.14$ ) we plot the coefficients $A^{(0)}$ (dotted curve) and $A^{(1)}$ (dash-dotted curve) for values of $\hat{k}$ close to $\bar{k}$. This diagram is given in support of the arguments that lead to the condition (Eq. (9)) for the onset of the instability.

relation cannot be taken to be small. As described previously, we have in mind two different kinds of situations of astrophysical interest. The case of counter-rotation is associated with $\hat{\delta} \approx-1$ so that we expect $\eta$ and $\Delta$ to have finite values. As noted earlier in the Introduction, this case has already been addressed in the literature. The second case, where the velocity difference of the two components, being generated by the asymmetric drift, is small, has been dealt with in the previous subsection; here, in order to consider $\eta=O(1)$, we should take $m$ to be large. In this new regime of open waves the basic dispersion relation Eq. (4) would be modified and there would exist additional destabilizing mechanisms (see Bertin et al. 1989; note the threshold value indicated by their Eq. (5.8), which, for a flat rotation curve, reduces to $Q^{2}=3$ ). For this, a consistent asymptotic analysis might be developed in an approach similar to the one followed in this paper, but for simplicity we omit such analysis in this paper.

In Figs. 3 and 4 we illustrate the qualitative behavior of the dispersion relation at fixed value of $\hat{\delta}$ for varying $\eta$ and for varying $m$ (with fixed $\Omega_{\mathrm{h}} / \kappa_{\mathrm{h}}$ ); in each plot the dashed and solid lines represent the imaginary and real part of the dimensionless frequency $v$, respectively. Since, at variance with the case of axisymmetric perturbations, the unstable root has a non-vanishing real part of the frequency, we are in the presence of a convective instability (see LJH97). Following the general arguments used by LJH97, we can also confirm for this general case of $\eta=O(1)$ that instability can develop when $\nu_{\mathrm{R}}\left(\nu_{\mathrm{R}}-\eta\right)<0$, i.e. when $\Omega_{\mathrm{h}}<\Omega_{\mathrm{p}}<\Omega_{\mathrm{c}}$.

In conclusion, the dispersion relation indicates the existence of unstable roots with dimensionless growth-rate (imaginary part of $v$ ) up to $0.1-0.2$ for small values of $m$, and formally up to 0.4 for higher values of $m$.

\subsection{Relevance to astrophysical applications}

As we will discuss further in the following section, the main context for astrophysical applications that we have in mind is that of normal spiral galaxies.

The strategy that we have used has been to focus on a condition of marginal stability with respect to axisymmetric perturbations in the two-component disk $\left(Q_{\mathrm{h}}=\bar{Q}(\alpha, \beta)\right)$ in order to demonstrate that the relative motion of the two components, independently of axisymmetric Jeans-type instabilities, is destabilizing. The instability that we have addressed can obviously occur under more general conditions than $Q_{\mathrm{h}}=\bar{Q}(\alpha, \beta)$, as shown 

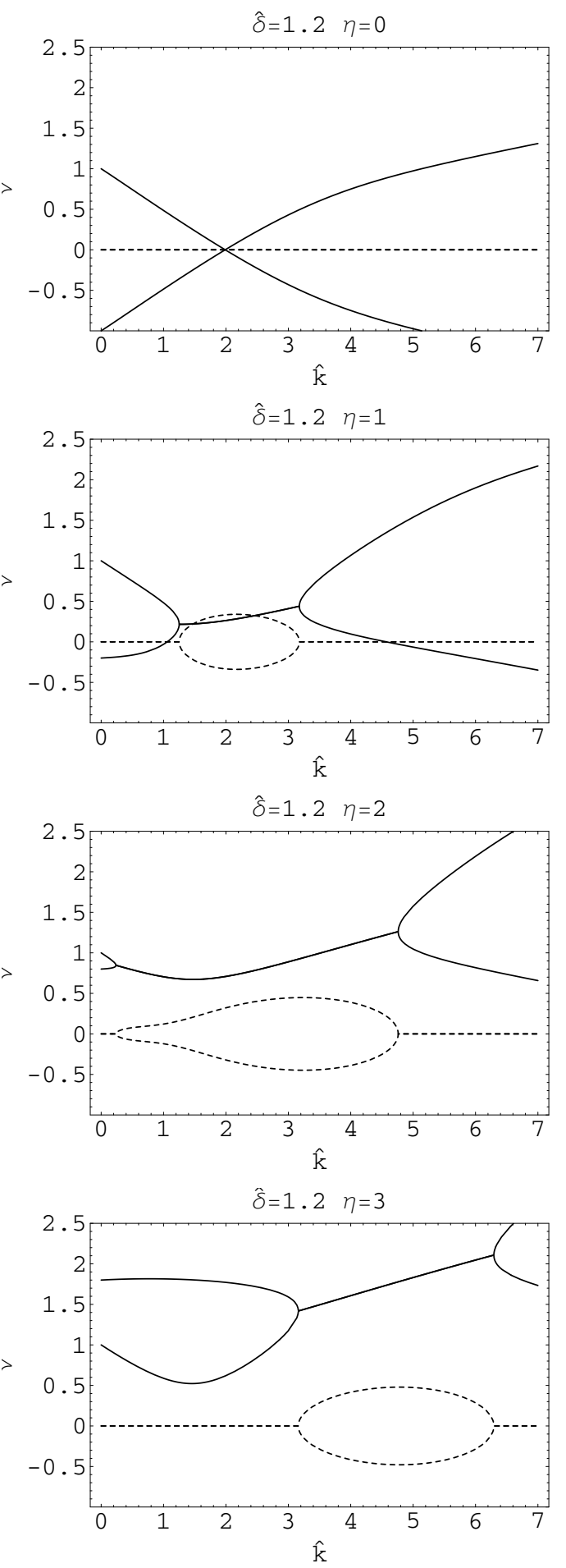

Fig. 3. The dispersion relation for varying $\eta$. In each frame the real (solid lines) and imaginary (dashed lines) part of $v$ are given as a function of the dimensionless wavenumber $\hat{k}$, for $\eta=0, \eta=1, \eta=2, \eta=3$ (from top to bottom). The other parameters have been set to the following values: $\hat{\delta}=1.2, \alpha=0.1, \beta=0.1, Q_{\mathrm{h}}=\bar{Q}\left(\alpha / \hat{\delta}^{2}, \beta / \hat{\delta}^{2}\right) \approx 1.14$.

in Fig. 5; in particular, instability can occur for $\alpha=0.1$ and $\beta=2.5 \times 10^{-2}$ (parameter values suggested by studies of the solar neighborhood) even for $Q_{\mathrm{h}}>1.5$.

The asymmetric drift instability, being driven by the relative motion of the two components, is expected to be ubiquitous and to coexist (as mentioned in the first paragraph of Sect. 3.3) with other sources of instability. As a result, the destabilizing effect
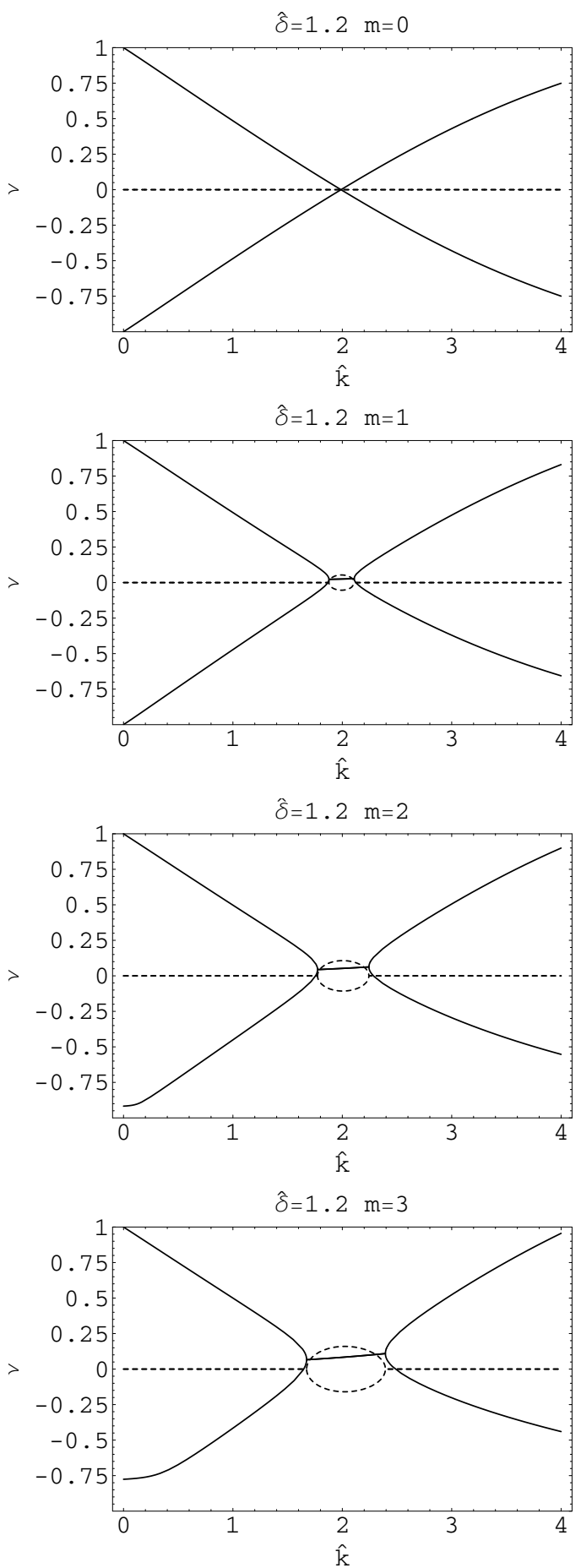

Fig. 4. The dispersion relation for varying $\mathrm{m}$. In each frame the real (solid lines) and imaginary (dashed lines) part of $v$ are given as a function of the dimensionless wavenumber $\hat{k}$, for $m=0, m=1, m=2$, $m=3$ (from top to bottom). The other parameters have been set to the following values: $\hat{\delta}=1.2, \alpha=0.1, \beta=0.1, Q_{\mathrm{h}}=\bar{Q}\left(\alpha / \hat{\delta}^{2}, \beta / \hat{\delta}^{2}\right) \approx 1.14$, with the additional condition of flat rotation curve $\Omega_{\mathrm{h}} / \kappa_{\mathrm{h}}=\sqrt{2} / 2$.

that we have discussed, even if present in realistic simulations of two-component disks, may be difficult to single out from other sources of instability and from a number of effects related to the specific modeling used in each simulation (e.g., see Orlova et al. 2002; see also Noh et al. 1991; Korchagin \& Theis 1999).

Interestingly, the instability described in this paper is expected to operate non-linearly to couple the two components (that is to keep cold and hot components under physical 

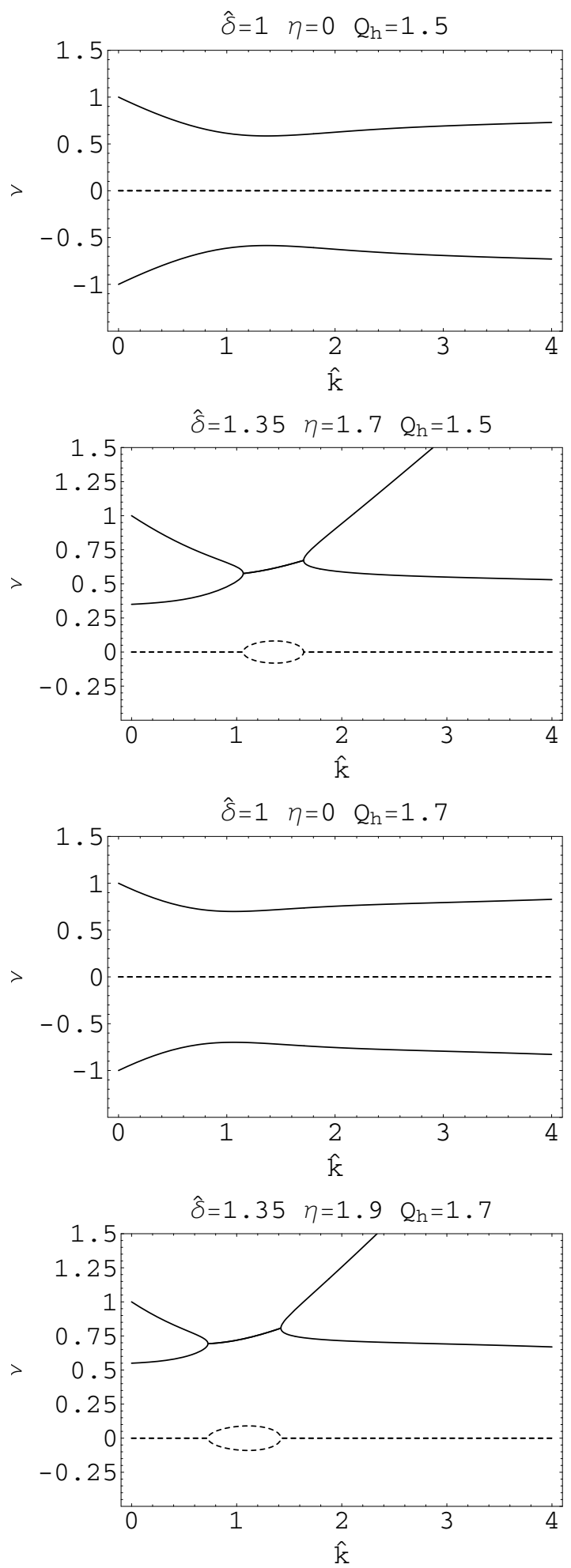

Fig. 5. The asymmetric drift instability for a disk stable with respect to axisymmetric instabilities, $Q_{\mathrm{h}}>\bar{Q}(\alpha, \beta)$. Here $\alpha=0.1, \beta=2.5 \times 10^{-2}$, values suggested by conditions in the disk of the Milky Way. The vertical axes in the second and fourth frames start from -0.50 to better illustrate the imaginary part of the root of the dispersion relation (Eq. (4)).

conditions not too far apart), counteracting the effects of the cooling processes present in the cold gas, which favor the decoupling of the two components. This makes the asymmetric drift instability participate in the "thermostat" of self-regulation (necessary for grand design spiral structure to be maintained, see Bertin \& Romeo 1988; Bertin et al. 1989). The natural emergence of self-regulation has been demonstrated recently in numerical experiments of the dynamics of self-gravitating protostellar disks (Lodato \& Rice 2004).

Another point that we wish to emphasize is that the study that we have performed is not limited to "small-scale instabilities" (the complex unstable root shown in Figs. 4 and 5 occurs at $\hat{k}$ of order unity, which does not correspond to "small scale" wavelengths); in fact, this property distinguishes the mechanism studied in this paper from the destabilizing effect on axisymmetric stability associated with the presence of cold gas, which indeed tends to act on the small scale.

\section{Discussion and conclusions}

In this paper we have shown that a self-gravitating thin disk made of a hotter and a cooler component can be unstable (with respect to non-axisymmetric density waves) because of the different amounts of asymmetric drift associated with the two components, even when the disk is stable in the sense of Jeans (with respect to axisymmetric density waves). The unified analysis performed here is based on a local dispersion relation from which we can easily recover the limits of previous stability investigations. In particular, we can recognize the two-component Jeans stability conditions, as studied by BR88, and the instability of counter-rotating disks, as discussed by LJH97 (in a technically more complicated study). For the case of small relative velocity between the two components, which is the focus of the present paper, and of low values of the azimuthal wavenumber $m$, the growth rate of the instability has been derived analytically. Here we aimed at investigating the asymmetric drift instability mechanism by itself and were not interested in assessing the stability or instability of a disk under specific physical conditions. For this latter purpose, it is well known that a number of other factors (such as the role of thickness; e.g., see Romeo 1992) should be properly taken into account. The collective effects realized in specific contexts will depend on the conditions of the basic state and on the non-linear phenomena that take place during evolution.

For applications to galaxy disks, the fluid-fluid analysis presented here is preliminary to a more realistic analysis in which the cold component is treated as a fluid, while the hotter component is treated in terms of the collisionless Boltzmann equation. Resonance effects, to be described by the equations of stellar dynamics, are expected to occur when the velocity difference between the two components is small, i.e. when it becomes comparable to the velocity dispersion of the stars associated with the hotter component; note that this condition refers to the velocity dispersion in the azimuthal direction, which is smaller by approximately a factor of $\kappa /(2 \Omega)$ than the radial velocity dispersion, which is responsible for the asymmetric drift. A proper description of resonances will lead to technical complications well beyond those addressed in the fluid-kinetic study of LJH97. Because of its physical origin, the asymmetric drift velocity is a priori bound to be relatively small, even for those galaxies (e.g., NGC 5064, NGC 3200, and NGC 2815) in which such velocity difference has been found to be, in magnitude, rather large (in the range of 50 to $100 \mathrm{~km} \mathrm{~s}^{-1}$; see Vega Beltrán et al. 2001; and Pizzella et al. 2004).

For galaxy disks, the instability studied in this paper is likely to induce turbulence and heating in the cold component and to contribute to the generation of a net inflow of material toward the center. In principle, one might think of a possible 
connection with the topic of extra-planar gas that is currently the subject of several investigations; this is the discovery that many galaxy disks, in addition to their "normal" thin HI layer, possess an "anomalous" thick HI component characterized by slower rotation (e.g., for NGC 2403, see Fraternali et al. 2002; for UGC 7321, see Matthews \& Wood 2003; for NGC 4559, see Barbieri et al. 2005). However, for these systems it is likely that the two HI components do not interpenetrate, but rather occupy different heights with respect to the equatorial plane; in this case, if instability occurs, the mechanism might be different from the one studied in this paper, and more in line with processes of the type investigated by Waxman (1979a,b).

Finding a mechanism able to act as a source of turbulence in disks brings us to consider possible applications to the longstanding issue of finding an adequate explanation for the anomalous viscosity that is thought to operate in accretion disks (see Shakura \& Sunyaev 1973). The present mechanism would contribute in those cases in which the various physical processes support the coexistence of two components at different levels of rotation around the central object. As a kind of two-stream instability, it might operate even for plasma disks.

This latter application naturally brings us to consider the case of protostellar disks. In fact, the mechanism studied in this paper appears to be the collective counterpart to the "viscous drag mechanism" (Weidenschilling 1977) that initially appeared to act against the picture of planet growth from planetesimals. Recent simulations have shown that such drag may actually have a beneficial role in the direction of planet formation (see Rice et al. 2004). This paper suggests that a specific collective process, independent of viscosity, may operate and contribute in a way similar to that of mechanisms present in viscous disks (see also Youdin \& Goodman 2005). The very simple analytical study provided in the present paper offers a description, at the linear level, of the collective instabilities underlying such interesting process.

Acknowledgements. We wish to thank Giuseppe Lodato for a number of interesting suggestions and Donald Lynden-Bell for useful conversations on the subject of this paper. This work was partially supported by MIUR of Italy (cofin2004).

\section{References}

Barbieri, C.V., Fraternali, F., Oosterloo, T., et al. 2005, A\&A, 439, 947

Bertin, G., \& Mark, J. W. K. 1980, A\&A, 88, 289

Bertin, G., \& Casertano, S. 1982, A\&A, 106, 274
Bertin, G., \& Romeo, A. B. 1988, A\&A, 195, 105 (BR88)

Bertin, G., Lin, C. C., Lowe, S. A., \& Thurstans, R. P. 1989, ApJ, 338, 104

Bertola, F., Cinzano, P., Corsini, E. M., et al. 1996, ApJ, 458, L67

Bettoni, D., Galletta, G., García-Burillo, S., \& Rodríguez-Franco, A. 2001, A\&A, 374, 421

Bottema, R. 1993, A\&A, 275, 16

Cava, A. 2004, Tesi di Laurea, Università di Milano

Comins, N. F., Lovelace, R. V. E., Zeltwanger, T., \& Shorey, P. 1997, ApJ, 484, L33

Fraternali, F., van Moorsel, G., Sancisi, R., \& Oosterloo, T. 2002, AJ, 123, 3124 Graham, R. 1967, MNRAS, 137, 25

Hunter, J. H., Whitaker, R. W., \& Lovelace, R. V. E. 1997, ApJ, 482, 853

Jog, C. J., \& Solomon, P. M. 1984a, ApJ, 276, 114

Jog, C. J., \& Solomon, P. M. 1984b, ApJ, 276, 127

Jore, K. P., Broeils, A. H., \& Haynes, M. P. 1996, AJ, 112, 438

Kato, S. 1972, PASJ, 24, 61

Krall, N. A., \& Trivelpiece, A. W. 1973, Principles of plasma physics (New York: McGraw-Hill)

Korchagin, V. I., \& Theis, Ch. 1999, A\&A, 347, 442

Lin, C. C., \& Shu, F. H. 1966, Proc. Natl. Acad. Sci. USA, 55, 229

Lodato, G., \& Rice, W. K. M. 2004, MNRAS, 351, 630

Lovelace, R. V. E., Jore, K. P., \& Haynes, M. P. 1997, ApJ, 475, 83 (LJH97)

Lynden-Bell, D. 1967, Lect. Appl. Math., 9, 131

Mandica, S. 2001, Tesi di Laurea, Università di Pisa

Mark, J. W.-K. 1976, ApJ, 206, 418

Marochnik, L. S., \& Suchkov, A. A. 1969, Ap\&SS, 4, 317

Marochnik, L. S., \& Suchkov, A. A. 1974, Uspekhi fiz. Nauk, 112, 275

Matthews, L. D., \& Wood, K. 2003, ApJ, 593, 721

Merrifield, M. R., \& Kuijken, K. 1994, ApJ, 432, 575

Miller, R. H., Prendergast, K. H., \& Quirk, W. J. 1970, ApJ, 161, 903

Nelson, A. H. 1976, MNRAS, 174, 661

Nelson, R. W., \& Tremaine, S. 1995, MNRAS, 275, 897

Noh, H., Vishniac, E. T., \& William, D. C. 1991, ApJ, 383, 372

Orlova, N., Korchagin, V., \& Theis, Ch. 2002, A\&A, 384, 872

Pizzella, A., Corsini, E. M., Vega Beltrán, J. C., \& Bertola, F. 2004, A\&A, 424, 447

Prada, F., Gutiérrez, C. M., Peletier, R. F., \& McKeith, C. D. 1996, ApJ, 463, L9 Quirk, W. J. 1971, ApJ, 167, 7

Rice, W. K. M., Lodato, G., Pringle, J. E., Armitage, P. J., \& Bonnell, I. A. 2004, MNRAS, 355, 543

Rix, H.-W., Franx, M., Fisher, D., \& Illingworth, G. 1992, ApJ, 400, L5

Romeo, A. B. 1992, MNRAS, 256, 307

Rubin, V. C., Graham, J. A., \& Kenney, J. D. P. 1992, ApJ, 394, L9

Saslaw, W. C. 1985, Gravitational physics of stellar and galactic systems (Cambridge: Cambridge University Press)

Sellwood, J. A., \& Carlberg, R. G. 1984, ApJ, 282, 61

Sellwood, J. A., \& Merritt, D. 1994, ApJ, 425, 530

Shakura, N. J., \& Sunyaev, R. A. 1973, A\&A, 24, 337

Sweet, P. A. 1963, MNRAS, 125, 285

Thakar, A. R., Ryden, B. S., Jore, K. P., \& Broeils, A. H. 1997, ApJ, 479, 702

Vega Beltrán, J. C., Pizzella, A., Corsini, E. M., et al. 2001, A\&A, 374, 394

Waxman, A. M. 1979a, ApJS, 41, 635

Waxman, A. M. 1979b, ApJS, 41, 647

Weidenschilling, S. J. 1977, MNRAS, 180, 57

Youdin, A. N., \& Goodman, J. 2005, ApJ, 620, 459 